Check for updates

Cite this: RSC Adv., 2018, 8, 4084

Received 18th October 2017

Accepted 18th December 2017

DOI: 10.1039/c7ra11491e

rsc.li/rsc-advances

\section{$\alpha$-Lipoic acid alleviates pentetrazol-induced neurological deficits and behavioral dysfunction in rats with seizures via an Nrf2 pathway}

\author{
Yahong Cheng, (D) a Fei Luo, ${ }^{a}$ Qianying Zhang, ${ }^{a}$ Ying Sang, (D) ${ }^{a}$ Xiaofang Chen, ${ }^{a}$ \\ Lu Zhang, ${ }^{a}$ Yonggang Liu, ${ }^{a}$ Xiaofei Li, ${ }^{a}$ Jian Li, ${ }^{a}$ Hong Ding ${ }^{\star a}$ and Yunqing Mei ${ }^{\star b c}$
}

Epilepsy (EP) is a type of chronic brain disease characterized by transient central nervous system malfunction which is the result of neuron paradoxical discharge in the brain. In a recent study, we evaluated the neuroprotective effect of $\alpha$-lipoic acid on pentetrazol-induced rats. Epileptic rats were induced by intraperitoneal injection of pentetrazol $\left(35 \mathrm{mg} \mathrm{kg}^{-1}\right)$, and were intragastrically administered with $\alpha$-lipoic acid for a month. The treatment with $\alpha$-lipoic acid significantly reduced the total frequency of epileptic seizures and improved the behavior impairment and cognitive disorder caused by pentetrazol toxicity. With the administration of $\alpha$-lipoic acid, not only did the apoptosis rate of nerve cells decrease, but also the formation of nitric oxide and malondialdehyde was inhibited. We also recorded evidence suggesting the treatment of $\alpha$-lipoic acid could enhance the activity of antioxidant enzymes. Furthermore, in the hippocampus area of the brain, $\alpha$-lipoic acid treatment promoted the translocation of Nrf2 in the nuclear fraction and increased the expression of HO-1 and NQO1. Our data indicated that $\alpha$-lipoic acid may be a potential candidate for lessening the number of epileptic attacks, improving the behavior and cognitive disorder and cutting down the apoptosis rate of nerve cells in a pentetrazol-induced rat model through activating the Nrf2 signaling pathway.

\section{Introduction}

Epilepsy is a chronic neurological disorder marked by spontaneous recurrent seizures. Epileptic seizures may lead to selective neuron injury and sometimes even neuron loss, resulting in changes of brain structure and brain function, such as the proliferation of glial cells and synaptic remodeling. ${ }^{1}$ The pathogenesis of epilepsy is very complex, and has not been fully elucidated. Epilepsy is triggered by the abnormal discharge of neurons, and this abnormal discharge can be induced by several factors, such as oxidative stress injury, excitatory toxicity of glutamate, intracellular calcium overload, etc. ${ }^{2}$ It is generally believed that a chain reaction of oxygen free radicals triggered by oxidative stress is the core that induces neuron damage. ${ }^{2}$ In addition, patients who suffer from epilepsy have a $30-40 \%$ probability of developing cognitive dysfunction, which will severely impact their lives. ${ }^{3}$ However, there is presently no

${ }^{a}$ Key Laboratory of Combinatorial Biosynthesis and Drug Discovery, Ministry of Education, Wuhan University School of Pharmaceutical Sciences, Wuhan University, Wuhan, Hubei, P. R. China. E-mail: dinghong1106@whu.edu.cn; Tel: $+8613007162084$

${ }^{b}$ Department of Cardio-Thoracic Surgery, Tongji University School of Medicine, Shanghai, China. E-mail: drmeiyq2004@tongji.edu.cn; Tel: +8602166111535

'Department of Cardio-Thoracic Surgery, Xiangyang No 1 People's Hospital, Hubei University of Medicine, Hubei, China effective therapeutic method for epilepsy treatment. ${ }^{4}$ Therefore, it is essential to find a new, adequate method for epilepsy therapy.

Pentetrazol, as a central stimulant, can cause neuronal apoptosis in the central nervous system (CNS), especially in the hippocampus area., ${ }^{5,6}$ After intraperitoneal injection of pentetrazol, rats show a series of behavioral abnormalities and neurological deficits. Researches have shown that the concentrations of hydrogen peroxide and nitric oxide are increased while the activities of antioxidant enzymes are decreased following pentetrazol administration in the cultured neurocytes and in a pentetrazol-exposed rat model. ${ }^{7}$ In addition, seizures result in significant increase of oxygen free radical (FR) so that the body is not in a position to remove all. Lots of FR can directly cause oxidative injury of macromolecular substances, such as lipid, protein and DNA, and then damage the cell membrane even other cell structures. ${ }^{\mathbf{8}, 9}$ Meanwhile, FR also can be expected to result in apoptosis by means of inhibiting the mitochondrial function. ${ }^{10}$ Therefore, oxidative stress may play an important role in the pathogenesis of brain injury inflicted by epilepsy.

NF-E2-related factor 2 (Nrf2), a key factor in cellular oxidative stress, regulates expression of peroxiredoxins and phase II detoxification enzymes by interacting with the antioxidant response element (ARE). ${ }^{11} \mathrm{Up}$ to now, it has been confirmed that more than 200 genes encoding endogenous protective proteins are adjusted via Nrf2 signaling pathway. ${ }^{12}$ They are the vital role 
A

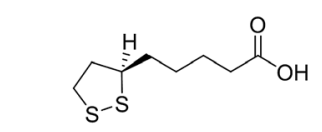

B

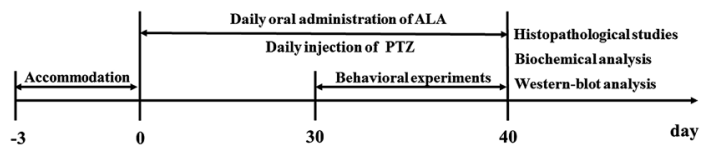

Fig. 1 (A) Chemical structure of ALA. (B) Schematic detailing the experiment arrangement for the time course of PTZ and ALA administration, and the timelines for the behavioral experiments, histopathological studies, biochemical analysis and western-blot analysis. (C) Mortality rates of the CON group, PTZ group and ALA group ( $N=$ $10)$. $\# \# p<0.01$ versus the CON group, $* * p<0.01$ versus the PTZ group.

in enhancing tissue ability of oxidation-resistance and protecting cells from toxic damage and have a unique pharmacological efficacy on anticancer and anti-apoptosis. ${ }^{13}$ Significantly, heme oxygenase 1 (HO-1) and NADPH: quinone oxidoreductase 1, NQO1 are two crucial antioxidant enzymes encoded by the pathway. ${ }^{14}$ The previous researches have revealed that activation of Nrf2-ARE pathway has important implications in preventing cerebral disease, such as Parkinson's disease, hemorrhage, cerebral infarction and head injury from neurological disorders. ${ }^{15}$ It indicates that Nrf2 expression plays a major role in neuroprotection. Thus, we speculate that up-regulating the Nrf2 pathway to enhance the response to oxidative injury may be a profitable epilepsy therapeutic conception.

$\alpha$-Lipoic acid (6,8-dithiooctanoic acid), a substance belonging to B-vitamins, is abundant in all kinds of food. ${ }^{16,17}$ The chemical structure formula of $\alpha$-lipoic acid is shown in Fig. 1A. $\alpha$-Lipoic acid exists in various parts of the body, and has a strong antioxidant ability, and it can reach the CNS easily through the blood-brain-barrier. ${ }^{16,18}$ It has received widespread attention of experts and academics because of its pharmacological values. $\alpha$-Lipoic acid can respond to oxidative stressinduced apoptosis and improve the cell viability. ${ }^{1,19}$ As studies have demonstrated, a promising candidate solution to protect the brain from neurons damage is linked with the activity of anti-oxidant enzymes.

At present, $\alpha$-lipoic acid shows great response to oxidative stress. However, there is no unequivocal evidence that indicates whether behavioral disorders and neurological dysfunction are associated with anti-oxidation. Therefore, in the present study, we evaluated the neuroprotective effect of $\alpha$-lipoic acid in both ethology and pathology for the first time. In addition, we investigated whether the function of $\alpha$-lipoic acid is related to the activation of Nrf2 signaling pathway.

\section{Materials and methods}

\subsection{Animals}

Male Sprague-Dawley rats (weighing 180-190 g, 6 weeks of age at the beginning of the experiments; Wuhan University
Laboratory Animal Center) were normally fed for 3 days. They were housed on a $12 \mathrm{~h}$ high-dark cycle at $25 \pm 2{ }^{\circ} \mathrm{C}$, and in a relative humidity of $60-80 \%$. Animals were fed on a diet of standard pellets and water. The animals were allowed to acclimate to the housing conditions for 3 days prior to experimentation. The animal study followed ARRIVE (Animal Research: Reporting In Vivo Experiments) guidelines and was approved by the Institutional Animal Care and Use Committee (IACUC), Wuhan University Center for Animal Experiment, Wuhan, China (AUP no. S2016110121).

\subsection{Epilepsy by pentetrazol and the experimental groups}

$\alpha$-Lipoic acid (Yongyao Biotechnology Co., Ltd, Taiyuan, China), dissolved in water, was given to rats through administration by gavage, with the concentrations for the in vivo experiments determined according to an unpublished study before in our laboratory. Pentetrazol was purchased from Macklin Reagent Co., Ltd (Shanghai, China). Random allocation was taken to averagely divide $30 \mathrm{SD}$ rats into three groups: normal control group (CON), pentetrazol-induced model group (PTZ) and $\alpha$ lipoic acid cure group (ALA). The handling methods of the groups were as follows: (i) $0.9 \%$ saline was administered by intraperitoneal injection in the normal control group. (ii) Pentetrazol-induced model group was injected with pentetrazol in a dose of $35 \mathrm{mg} \mathrm{kg}^{-1}$ for 30 days until the rats suffered from epileptic seizures at the level of 4-5 (according to the Racine scale). ${ }^{20}$ (iii) $\alpha$-Lipoic acid cure group was injected the same amount of pentetrazol intoxication. Besides, the rats were administered $20 \mathrm{mg} \mathrm{kg}^{-1} \alpha$-lipoic acid for a month. The determination of lipoic acid dose was based on our preliminary studies which revealed that lipoic acid at this dose improved symptoms of epilepsy better than at the dose of $10 \mathrm{mg} \mathrm{kg}^{-1}$ and had less side effect, such as poor compliance, than at the dose of $30 \mathrm{mg} \mathrm{kg}^{-1}$. Animals were euthanized with an overdose of isoflurane and hippocampal tissues were excised, weighed, snap frozen in liquid nitrogen, and stored in a $-80{ }^{\circ} \mathrm{C}$ freezer for histopathology and western blot analysis at the end of the experiment. The timetable of the experiment is shown in Fig. 1B.

\subsection{Mortality rate}

To monitor the survival situation of the animals $(n=10)$, the number of deaths was recorded at the end of the experiment.

\subsection{Seizure stage observation}

By peritoneal injection of pentetrazol for epilepsy, seizure stage was observed and scored based on a modified Racine scale, which is as follows:

Level 0: no response;

Level 1: rats present symptoms such as dazing and ear or face switching;

Level 2: animals appear behavior of whole body twitching without falling down or forelegs lifting;

Level 3: rats raise one or both sides of the forelimb but do not fall;

Level 4: animals develop symptom of falling on one side.

Level 5: rats suffer from generalized tonic clonic seizures. ${ }^{21}$ 


\subsection{Open field test}

The open-field test was conducted in a quiet room between 8:00 am and 13:00 pm as described previously. ${ }^{22}$ The open-field instrument (square area, $100 \mathrm{~cm} \times 100 \mathrm{~cm}$, high $50 \mathrm{~cm}$ ) was marked with a grid dividing it into 25 equal-sized squares. Each rat was positioned in the central square, initially adapting to the environment for $1 \mathrm{~min}$ and then observed for $5 \mathrm{~min}$. Scores of every animal were calculated by the number of rearing (defined as standing upright on its hind legs), the number of crossing (grid lines it crossed with at least three legs) and the dwell times in the central grids.

\subsection{Morris water maze}

The Morris water maze (MWM) test ${ }^{23}$ apparatus was a drumshaped pool (diameter: $160 \mathrm{~cm}$; high: $55 \mathrm{~cm}$ ) filled with water to a depth of $25 \mathrm{~cm}$ maintained at $25 \pm 0.5{ }^{\circ} \mathrm{C}$. The maze was divided into four quadrants (quadrant I, II, III, and IV) marked with different shapes on the walls. An escape platform (diameter: $10 \mathrm{~cm}$; high: $23 \mathrm{~cm}$ ) was submerged $1 \mathrm{~cm}$ under the water surface in the center of quadrant II. The MWM included two tests: the navigation task and the probe trial task.

Navigation task. The rat was gently lowered into the pool in different quadrants and allowed to swim freely to locate the platform within $60 \mathrm{~s}$. The latency to locate the platform was recorded. If time is more than $60 \mathrm{~s}$, then the rat will be placed on the platform for $15 \mathrm{~s}$, and the latency is $60 \mathrm{~s}$. Each animal performed four training trials daily for four consecutive days.

Probe trial task. A day after the last training trial, the animals underwent a probe trial session after the platform was removed, wherein they were allowed to swim for $60 \mathrm{~s}$.

\subsection{Histopathological studies}

The hippocampus tissues were immersed in $4 \%$ paraformaldehyde for $4 \mathrm{~h}$, and transferred to $70 \%$ ethanol. Individual lobes of the hippocampus tissues biopsy material were placed in processing cassettes, dehydrated through a serial alcohol gradient, and embedded in paraffin wax blocks. Before staining, $4 \mu \mathrm{m}$-thick tissue sections were dewaxed in xylene, rehydrated through decreasing concentrations of ethanol, and washed in PBS, and then stained with hematoxylin and eosin (H\&E). After staining, sections were dehydrated through increasing concentrations of ethanol and xylene, observed under a microscope to identify histopathological changes in the hippocampus area. ${ }^{24}$ Photographs of each of the slides were taken at $100 \times$ magnification.

\subsection{Immunohistological staining}

The hippocampus tissues were fixed in $4 \%$ paraformaldehyde for $48 \mathrm{~h}$, routinely embedded in paraffin. Sections of $4 \mu \mathrm{m}$ thickness were made using a microtome and were washed in PBS and permeabilized with $0.1 \%$ Triton X-100 citrate for $2 \mathrm{~min}$ on ice. TUNEL (TdT mediated dUTP Nick End Labelling) assay was performed using a kit from Roche (Mannheim, Germany), following the manufacture's instructions. Sections were incubated with Hoechst for nuclei staining. ${ }^{25}$ The percentage of apoptotic cells was calculated under a fluorescent microscope $(400 \times)$ from the ratio between TUNEL positive cells and total number of cells (nuclei staining with Hoechst).

\subsection{The measurement of SOD, MDA, CAT and NO}

After the behavioral tests, four rats selected randomly from each group were euthanized and hippocampus tissue was extracted in a cold environment. Then, tissues were added to cold saline at a ratio of 1 : 9 (weight of the tissue to volume of the saline), homogenized rapidly and centrifuged at $3000 \mathrm{rpm}$ for $15 \mathrm{~min}$ at $4{ }^{\circ} \mathrm{C}$. The supernatant was gathered for biochemical analysis. Superoxide dismutase (SOD), malondialdehyde (MDA), catalase (CAT) and nitric oxide (NO) were identified as four major biomarkers of oxidative stress. ${ }^{26}$ The activities of SOD and CAT, and the level of MDA were estimated with reagent kits bought from the Institute of Biological Engineering of Nanjing Jiancheng (Nanjing, China). NO production was measured by the Griess method according to the indication on NO assay kit purchased from Beyotime Biotechnology Co., Jiangsu, China. The assays were performed following the instruction of the test kits.

\subsection{Western-blot analysis}

Samples of hippocampal tissues isolated from the brain $(n=4)$, weighing $50 \mathrm{mg}$, were homogenized rapidly with cytoplasmic extraction reagent (CER) (10\%, w/v). Lysis buffer (Beyotime, Shanghai, China) was used to lyse tissues. The supernatants were collected and centrifuged at $12000 \mathrm{~g}$ for $10 \mathrm{~min}$ at $4{ }^{\circ} \mathrm{C}$. The cytoplasmic protein was gained after immediately drawing the supernatant to a pre-cooling Eppendorf tube. Completely absorbed the remaining supernatant, the pellet, added twice the volume of the nucleus extraction reagent (NER), was applied to extract the nuclear protein for evaluating the Nrf2 expression. Cytoplasmic and nuclear proteins were extracted with a reagent kit purchased from Boster Biotechnology Co., Wuhan, China. The concentrations of protein were measured using a BCA kit bought from Beyotime Biotechnology Co., Jiangsu, China. ${ }^{27}$ Equal amounts of proteins were submitted to western blot analysis. As much as $20 \mu \mathrm{g}$ of proteins were isolated by SDSPAGE, and then transferred onto the PVDF membrane. After being blocked with $5 \%$ bovine serum albumin, the PVDF membranes were incubated using primary antibodies rabbit anti-HO-1 (1 : 500, Boster Biotechnology, Wuhan, China), rabbit anti-NQO1 ( $1: 1000$, Proteintech Group, Inc., Chicago, USA), rabbit anti-Nrf2 (1: 500, Boster Biotechnology, Wuhan, China), and rabbit anti- $\beta$-actin $(1: 2000$, Google Biotechnology, Wuhan, China). In addition, specific nuclear rabbit anti-lamin B1 (ref. 28) (1:500, Boster Biotechnology, Wuhan, China) was incubated for estimating Nrf2 expression in the nucleus. After three washes with TBS-T, the blots were incubated with goat antirabbit IgG peroxidase conjugated secondary antibody ( 1 : 5000, Biosharp, Wuhan, China) for $1 \mathrm{~h}$. The blots were washed again, and then a chemiluminescent autographic assay was performed using chemiluminescence reagents (Beyotime, Biotechnology, Wuhan, China) to quantify the relative 
expression. Finally, the signal were observed on an X-ray film, and data analysis was performed using ImageJ software.

\subsection{The effects of $\alpha$-lipoic acid on normal animals}

We studied the effects of $\alpha$-lipoic acid on normal animals. The animals were administered with $\alpha$-lipoic acid for 3 days. Then, the rats were euthanized and the brains utilized for western blot analysis. The expressions of HO-1 and NQO1 as well as Nrf2 in the cytoplasm and nucleus were measured.

\subsection{Statistical analysis}

Statistical analysis was performed using SPSS 19.0 software. The data were expressed as mean $\pm \mathrm{SD}$. Data for the seizure score and escape latencies during the training were analysed with two-way analysis of variance (ANOVA). Measurement data of the other results were compared with single factor analysis of variance. Significance of variable comparisons between groups was determined by $t$-test. $p<0.05$ was considered to be statistically significant. All statistical analysis and graphs were performed using Graphpad Prism5 software (CA, USA).

\section{Results}

\subsection{Reduction effect of $\alpha$-lipoic acid on the death rate}

The death rate of each group was recorded, as shown in Fig. 1C. In the pentetrazol-induced model group, the mortality rate was $30 \%$, while it was $10 \%$ in the $\alpha$-lipoic acid cure group, which suggested $\alpha$-lipoic acid intervention significantly decreased the mortality rate caused by pentetrazol toxicity. And there was not any death in the CON group. The results implied that the rats' mortality rate increased considerably when injected with pentetrazol among these groups, especially in comparison with the control group. However, the death rate showed a noticeable decrease when administrated with $\alpha$-lipoic, which inhibited the death of the animals caused by pentetrazol induction.

\subsection{Decline by $\alpha$-lipoic acid on frequency of seizures}

After injected with pentetrazol, the rats presented symptoms such as dazing at the beginning, and then suffered from body twitching, and finally showed stiff-clonic onset. Seizure stage was evaluated via a Racine scale. In the PTZ group, the average attack level demonstrated upgrade tendency, and reached a peak of level 5 in day 30. The level of epileptic seizures indicated a significant decline after administrated with $\alpha$-lipoic acid compared with the PTZ group, and rats did not appear seizures in the control group (Fig. 2).

\subsection{Enhancement by $\alpha$-lipoic acid on animal exploration behavior}

The parameters evaluated in the open field were significantly different by $\alpha$-lipoic acid or pentetrazol treatments; total crossing in the open field, percent of center crossing of open field, and time spent at the center of the open field using oneway ANOVA. $\alpha$-Lipoic acid increased total crossing. Pentetrazol toxic rats were inclined to travel around the box in comparison

\section{Seizure score}

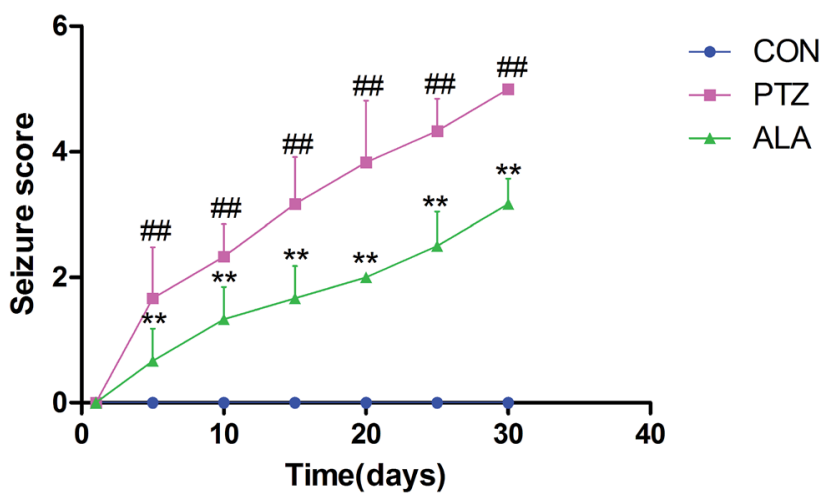

Fig. 2 Effects of $\alpha$-lipoic acid treatment on mean seizure score in PTZ-induced kindling in rats. The two-way ANOVA for seizure score indicated a significant group-by-day interaction. All data were expressed as mean $\pm S D(N=10)$, \#\#p $<0.01$ versus the CON group, $* * p<0.01$ versus the PTZ group.

with the CON group. Consequently, the proportion of center crossing and the time spent at the center of the PTZ group were significantly less than those of the control group. However, under the treatment of $\alpha$-lipoic acid, the exploring behavior of the animals enhanced with an aggrandizement in locomotive activity (Fig. 3).

\subsection{Effect of $\alpha$-lipoic acid on spatial cognition and memory}

The Morris water maze test was implemented to assess the influence of $\alpha$-lipoic acid on spatial learning and memory, as shown in Fig. 4A-E. The PTZ group revealed an enhanced escape latency than the CON group throughout the training days, peculiarly on the ultimate training day. With the training going on, the ALA group proved a significantly shorter escape latency than that of the PTZ group (Fig. 4A). A probe trial task was carried out to evaluate how well the rats were aware of the location of the platform in the maze. The traveled distance, time and percent of time spent swimming in the target area of the PTZ group in the probe test was significantly reversed by treatment of $\alpha$-lipoic acid (Fig. 4B, C and E). Statistics proved that the times of crossings of the former location of the platform significantly raised by administration of $\alpha$-lipoic acid (Fig. 4D). Evidence exhibited that pentetrazol toxicity weakened the rat's
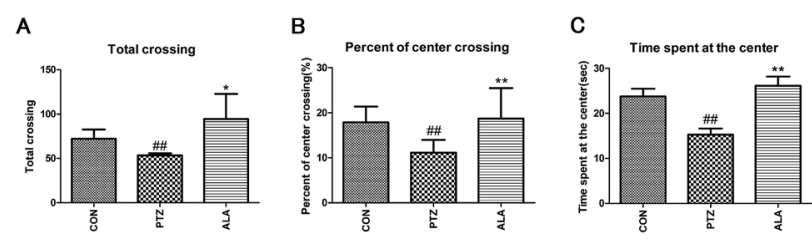

Fig. 3 Locomotive influence of pentetrazol intoxication and $\alpha$-lipoic acid treatment were elaborated by open-field test. (A) The number of total crossing in the open field. (B) Percent of center crossing of open field. (C) Time spent at the center of open field. All data were expressed as mean $\pm \mathrm{SD}(N=7), \# \# p<0.01$ versus the CON group, $* p<0.05, * * p$ $<0.01$ versus the PTZ group. 

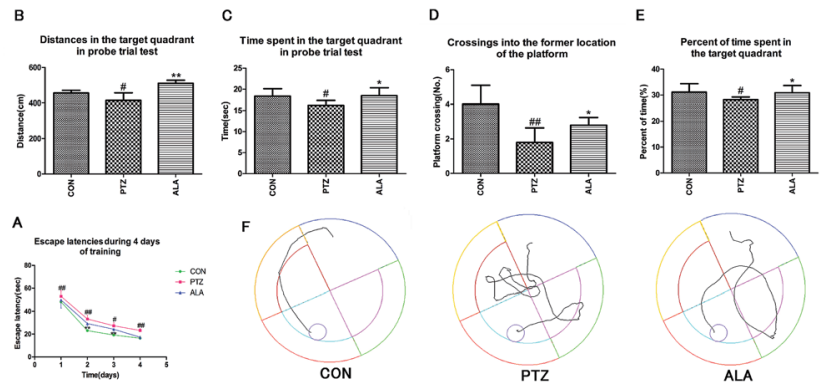

Fig. 4 Function of $\alpha$-lipoic acid on pentetrazol-induced spatial cognition and memory impairment by the Morris water maze test. (A) Two-way ANOVA for escape latencies shown as mean value of each trail session for four sequential days. (B) The distances traveled in the target quadrant during the probe trial task. (C) The time spent in the target quadrant during the MWM probe trial task. (D) The crossing into the former location of the submerged platform during the probe trial test. (E) The percent of time spent in the target quadrant in the MWM probe trial task. (F) Swimming tracks recorded via a video tracking camera system. All data were expressed as mean $\pm \operatorname{SD}(N=7)$. \#p< $0.05, \# \# p<0.01$ versus the CON group, $* p<0.05, * * p<0.01$ versus the PTZ group.

ability for spatial learning and memory, which were changed for the better via the treatment of $\alpha$-lipoic acid (Fig. 4E).

\subsection{Influence of $\alpha$-lipoic acid on oxidative stress and phase II detoxification}

To estimate the effect of $\alpha$-lipoic acid on oxidative stress, the SOD and CAT activities were measured as well as the MDA and NO levels. The result showed that $\alpha$-lipoic acid significantly reduced the level of MDA and NO and aggrandized the activities of the anti-oxidant enzymes, which included the activities of SOD and CAT, as shown in Fig. 5A-D. According to the protein expression levels of HO-1 and NQO1, $\alpha$-lipoic acid might largely enhance the reaction of phase II detoxification. The expression levels of HO-1 and NQO1 are shown in Fig. 5D-F, where the quantity of protein expression declined significantly after PTZ treatment for 30 days. However, compared with the PTZ group, the ALA group revealed a higher level of protein expression.

\subsection{Effect of $\alpha$-lipoic acid on neuronal damage and neuronal apoptosis}

Effects of $\alpha$-lipoic acid on neuronal damage and apoptosis were evaluated after behavioral experiments. Hematoxylin-eosin staining was employed to estimate the neuronal status. Histological observations of hippocampus sections from the normal control group showed normal cellular architecture. In contrast, the PTZ group manifested the most severe damage among all groups. Brain sections exhibited deflation of cell volumes, nuclear condensation, cell decrease and disorganization, especially in CA1 and CA3 areas. However, $\alpha$-lipoic acid could significantly reverse the nerve cell damage (Fig. 6A). To proof whether $\alpha$-lipoic acid $20 \mathrm{mg}$ per $\mathrm{kg}$ treatment adjusted apoptosis and apoptotic markers, TUNEL was utilized. The consequence revealed that $\alpha$-lipoic acid $20 \mathrm{mg}$ per kg administration significantly reduced the amount of TUNEL-positive
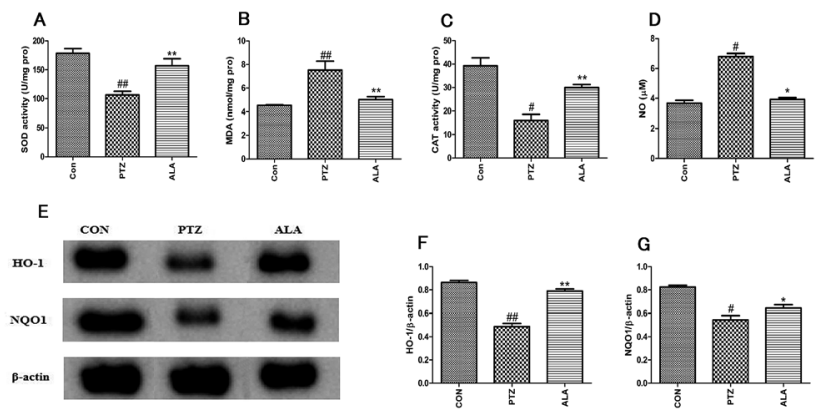

Fig. 5 Influence of $\alpha$-lipoic acid on oxidative stress and phase II detoxifying. The SOD activities (A), MDA level (B), CAT activities (C) and $\mathrm{NO}$ level (D) in the experiments are shown. (E) Representative western blotting for HO-1 and NQO1 expression of the CON, PTZ and ALA groups. (F) Densitometric value of the $\mathrm{HO}-1$ protein bands normalized to the respective $\beta$-actin. (G) Densitometric value of the NQO1 protein bands normalized to the respective $\beta$-actin. All data were expressed as mean $\pm \operatorname{SD}(N=7)$. \#p $<0.05$, \#\#p $<0.01$ versus the CON group, $* p<$ $0.05, * * p<0.01$ versus the PTZ group.

cells in the hippocampus area of the brain in comparison with the model group in Fig. 6B and $\mathrm{C}(p<0.01)$.

\section{7 $\alpha$-Lipoic acid potently regulates the Nrf2 signal pathway}

To explore the role of Nrf2 signal pathway in the neuroprotective effect of $\alpha$-lipoic acid against epilepsy, we detected the protein expression of Nrf2 both in the nuclear fraction and the cytosolic fraction from the hippocampus. It was found that the level of

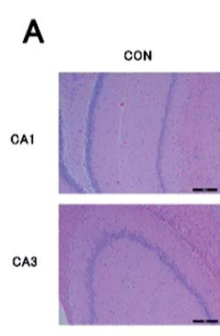

B
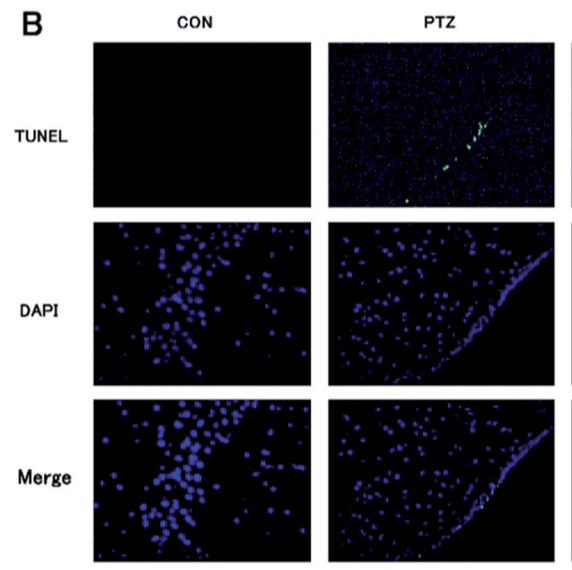

C
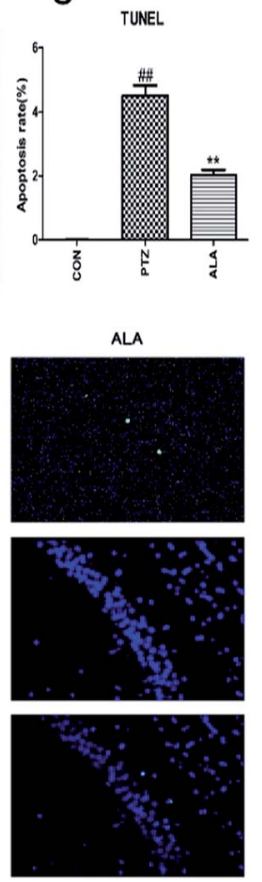

Fig. 6 Effects of $\alpha$-lipoic acid on the neuronal damage (HE staining) (A) and apoptosis (TUNEL) (B and C). All data were expressed as mean $\pm \mathrm{SD}(N=4) . \# \# p<0.01$ versus the CON group, ${ }^{* *} p<0.01$ versus the PTZ group. 

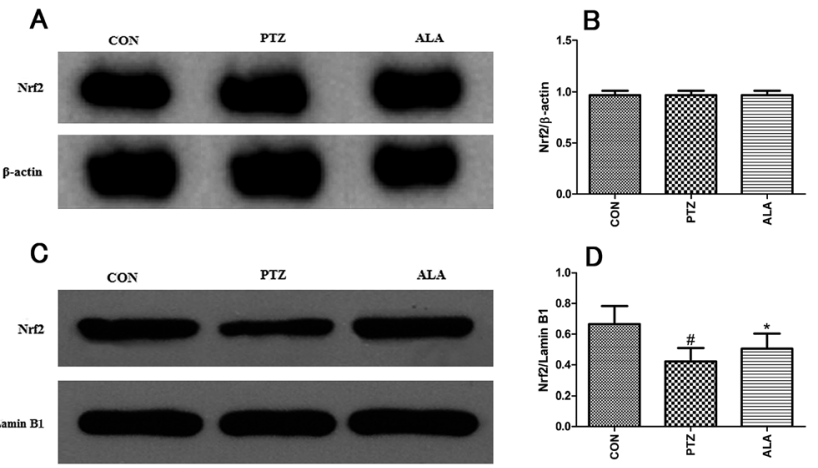

Fig. 7 The effect of $\alpha$-lipoic acid on the protein expression of Nrf2 in the nuclear and cytosolic fractions analyzed by western blotting. (A) Representative western-blot for the Nrf2 protein in the cytosolic fraction. (B) Densitometric value of the Nrf2 protein bands normalized to the respective $\beta$-actin. (C) Representative western-blot for the Nrf2 protein in the nuclear fraction. (D) Densitometric value of the Nrf2 protein bands normalized to the respective Lamin B1. All data were expressed as mean $\pm \mathrm{SD}(N=7)$. $\# p<0.05$ versus the CON group, $* p<$ 0.05 versus the PTZ group.

Nrf2 protein expression in cytosolic fraction was the same as the normal level. And the level of Nrf2 protein expression in the cytosolic fraction basically kept unchanged (Fig. 7A and B). However, Nrf2 nuclear translocation in the PTZ group was declined while $\alpha$-lipoic acid administration significantly enhanced Nrf2 expression level in the nuclear fraction (Fig. 7C and D). As previously predicted, $\alpha$-lipoic acid treatment also raised the expression of HO-1 and NQO1 in the hippocampus, two targets of the Nrf2. Therefore, $\alpha$-lipoic acid treatment may result in accelerating the nuclear translocation of Nrf2 via regulating the Nrf2 signal pathway.
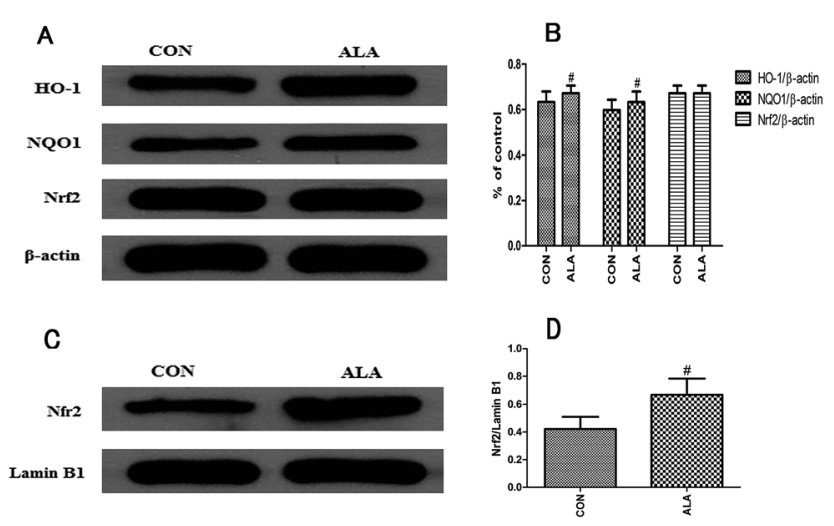

Fig. 8 Expression level of $\mathrm{HO}-1, \mathrm{NQO} 1$ and Nrf2 nuclear translocation in normal brain after administering $\alpha$-lipoic acid for 3 days. (A) Representative western blotting for Nrf2 protein in the nuclear fraction, and $\mathrm{HO}-1$ and NQO1 expression of the CON and ALA groups. (B) Densitometric value of the HO-1, NQO1 and Nrf2 protein bands normalized to the respective $\beta$-actin. (C) Representative western-blot for the Nrf2 protein in the nuclear fraction. (D) Densitometric value of the Nrf2 protein bands normalized to the respective Lamin B1. All data were expressed as mean $\pm \mathrm{SD}(N=7)$. $\# p<0.05$ versus the CON group.

\section{8 $\alpha$-Lipoic acid enhances Nrf 2 expression in normal brains}

To clarify whether the neuroprotective function of $\alpha$-lipoic acid was related to a defensive stimulation of anti-oxidative response, normal rats were intragastrically treated $\alpha$-lipoic acid for 3 days. Then, we evaluated the expression levels of HO-1 and NQO1, and Nrf2 nuclear translocation as well. Fig. 8A and B indicated that $\alpha$-lipoic acid significantly enhanced the expression of HO-1 and NQO1 in the normal brain, and the protein expression level of Nrf2 in the cytosolic fraction kept unchanged compared with the normal brains. Besides, $\alpha$-lipoic acid increased the accumulation of $\mathrm{Nfr} 2$ in the nuclear fraction, as Fig. 8C and D show.

\section{Discussion}

The present research provides support for investigation of Nrf2 signal pathway regulation by $\alpha$-lipoic acid as a novel device to ameliorate nerve damage and reduce the total frequency of epileptic seizures. In our study, we obtain the results that: (1) $\alpha$ lipoic acid treatment protects animals against epileptic seizures; (2) $\alpha$-lipoic acid sufficiently improves the rats' motor dysfunction and cognitive disorder induced by pentetrazol toxicity; (3) $\alpha$-lipoic acid efficiently defends the brain tissues from oxidative injury; (4) the neuroprotective effect of $\alpha$-lipoic acid on animals is related to the Nrf2 signal pathway.

Pentetrazol results in epileptic seizures and the pentetrazol model is one of the most widely used models to assess epilepsy pathogenesis and therapeutic interventions. ${ }^{29,30}$ In our research, the pentetrazol-induced model was used to investigate the pathology of epilepsy caused by neuronal apoptosis in the hippocampus area. Researches have shown that seizures lead to significant increase of reactive oxygen species. ${ }^{31}$ As a result, the brain is affected by neuronal apoptosis due to oxygen free radical inhibiting the mitochondrial function. ${ }^{32}$ Studies indicate that the activities of the anti-oxidant enzymes such as catalase, GPx, and total SOD are decreased by pentetrazol toxicity. ${ }^{33,34}$ In our present study, $\alpha$-lipoic acid significantly reduced the level of MDA and NO and aggrandized the activities of the anti-oxidant enzymes, which included the activities of SOD and CAT. Thus, we speculate that $\alpha$-lipoic acid may alleviate epileptic seizures caused by pentetrazol intoxication via fighting against oxidative stress.

Nrf2, interacting with the anti-oxidative response element regions, could regulate expression of peroxiredoxins and phase II detoxification enzymes. ${ }^{12}$ Stimulation of the Nrf2 signal pathway is a hopeful therapeutic approach in curing neurodegenerative diseases. ${ }^{13}$ The previous researches have demonstrated that activation of Nrf2 pathway plays a significant role in preventing cerebral disease, such as Parkinson's disease, hemorrhage, cerebral infarction and head injury from neurological disorders. ${ }^{35}$ It is reported that Nrf2 signal pathway is beneficial to the implementation of a promising method for defending nerve cells from oxidation, inflammation and accelerating autophagy. ${ }^{36,37}$ Therefore, activation of Nrf2 pathway is regarded as an accessible way to treat epilepsy by responding to oxidative damage. 
As reported, $\alpha$-lipoic acid has a high resistance to oxidative stress to protect various types of cells from apoptosis. ${ }^{38}$ Studies have shown that $\alpha$-lipoic acid facilitated induced Nrf2-mediated ARE pathway activation and induced Nrf2-mediated HO-1 in human retinal pigment epithelium. ${ }^{15}$ In addition, current therapeutic method of epilepsy might depend on up-regulating Nrf2 protein expression. ${ }^{39,40}$ Under the general physiological conditions, Nrf2 exists in the cell plasma, linked to the Kelchlike ECH-associated protein 1 (Keap1). ${ }^{41}$ The structure is too unstable to be rapidly degraded by the ubiquitin-proteasome pathway. When it happens in oxidative stress, the correlation breaks down and leads to Nrf2 accumulation in the cell nucleus. Nrf2 in the cell nucleus is linked to small molecules such as Maf to constitute a heterodimer, which is combined with the antioxidant response element (ARE) to activate the expression of downstream target genes (HO-1 and NQO1) functionally protecting the cells from injury. ${ }^{42,43}$ In this research, we found that $\alpha$-lipoic acid significantly improved the behavior and cognitive disorder of pentetrazol-induced rats. Therefore, $\alpha$-lipoic acid may be a potential candidate to help to treat epilepsy via activating Nrf2 nuclear translocation.

It is generally accepted that a chain reaction of oxygen free radical triggered by oxidative stress is the core that induces neuron damage. ${ }^{44}$ One of the hopeful therapeutic treatments for neuroprotection cure is inhibiting oxidative stress by activating the Nrf2 signaling pathway in nerve cells or tissues. ${ }^{45}$ Epilepsy is a chronic neurological disorder characterized by spontaneous recurrent seizures. ${ }^{46}$ In addition, the patients who suffer from epilepsy have a very high probability to get cognitive dysfunction induced by hippocampus ruin. ${ }^{47}$ The pathogenesis of epilepsy is very complex, and has not been fully elucidated. Previous study has demonstrated that the level of Nrf2 expression is closely associated with epileptic seizures. When the cells are stimulated by various exogenous stimulus such as oxidative stress, Nrf2 is separated from Keap1, and then transferred into the nucleus to combine with the ARE, as a result, inducing the transcription of the antioxidant genes. ${ }^{39} \alpha$-Lipoic acid treatment promoted the translocation of Nrf2 in the nuclear fraction and increase the expression levels of HO-1 and NQO1. ${ }^{48} \alpha$-Lipoic acid, a sulfur-containing C8 fatty acids, is one of the powerful antioxidants ever discovered whose antioxidant capacity is 400 times than vitamin C. ${ }^{16}$ Furthermore, $\alpha$-lipoic acid can upregulated the Nrf2 signal pathway. Therefore, apoptosis induced by oxidative stress could be suppressed by $\alpha$-lipoic acid via activating the Nrf2 signal pathway.

In a word, our experiments demonstrated that $\alpha$-lipoic acid could lessen the number of epileptic attacks and improve the behavior and cognitive disorder in pentetrazol toxic rats. We indicated that $\alpha$-lipoic acid cuts down the apoptosis rate of nerve cells and ameliorates neurological deficits by upregulating the expression level of Nrf2. In addition, our study suggested that $\alpha$-lipoic acid might be a potential candidate for neuroprotection in the therapy of epilepsy.

\section{Conflicts of interest}

There are no conflicts to declare.

\section{References}

1 D. C. Tarquinio, W. Hou, A. Berg, W. E. Kaufmann, J. B. Lane, S. A. Skinner, K. J. Motil, J. L. Neul, A. K. Percy and D. G. Glaze, Longitudinal course of epilepsy in Rett syndrome and related disorders, Brain, 2017, 140, 306-318.

2 J. Sun, X. Gao, D. Meng, Y. Xu, X. Wang, X. Gu, M. Guo, X. Shao, H. Yan, C. Jiang and Y. Zheng, Antagomirs Targeting MiroRNA-134 Attenuates Epilepsy in Rats through Regulation of Oxidative Stress, Mitochondrial Functions and Autophagy, Front. Pharmacol., 2017, 8, 524.

3 U. P. Kundap, Y. Kumari, I. Othman and M. F. Shaikh, Zebrafish as a Model for Epilepsy-Induced Cognitive Dysfunction: A Pharmacological, Biochemical and Behavioral Approach,, Front. Pharmacol., 2017, 8, 515.

4 P. Bottger, S. Glerup, B. Gesslein, N. B. Illarionova, T. J. Isaksen, A. Heuck, B. H. Clausen, E. M. Fuchtbauer, J. B. Gramsbergen, E. Gunnarson, A. Aperia, M. Lauritzen, K. L. Lambertsen, P. Nissen and K. Lykke-Hartmann, Glutamate-system defects behind psychiatric manifestations in a familial hemiplegic migraine type 2 disease-mutation mouse model, Sci. Rep., 2016, 6, 22047.

5 S. Choopankareh, F. Vafaee, M. N. Shafei, H. R. Sadeghnia, R. Salarinia, L. Zarepoor and M. Hosseini, Effects of melatonin and theanine administration on pentylenetetrazole-induced seizures and brain tissue oxidative damage in ovariectomized rats, Turk. J. Med. Sci., 2015, 45, 842-849.

6 B. Zhang, J. W. Zhang, W. P. Wang, R. F. Dong, S. Tian and C. Zhang, Effect of lamotrigine on epilepsy-induced cognitive impairment and hippocampal neuronal apoptosis in pentylenetetrazole-kindled animal model, Synapse, 2017, $71,2$.

7 X. Zhu, J. Dong, K. Shen, Y. Bai, Y. Zhang, X. Lv, J. Chao and H. Yao, NMDA receptor NR2B subunits contribute to PTZkindling-induced hippocampal astrocytosis and oxidative stress, Brain Res. Bull., 2015, 114, 70-78.

8 S. Rowley, L. P. Liang, R. Fulton, T. Shimizu, B. Day and M. Patel, Mitochondrial respiration deficits driven by reactive oxygen species in experimental temporal lobe epilepsy, Neurobiol. Dis., 2015, 75, 151-158.

9 S. J. Chou, W. L. Tseng, C. T. Chen, Y. F. Lai, C. S. Chien, Y. L. Chang, H. C. Lee, Y. H. Wei and S. H. Chiou, Impaired ROS Scavenging System in Human Induced Pluripotent Stem Cells Generated from Patients with MERRF Syndrome, Sci. Rep., 2016, 6, 23661.

10 J. Guevara-Campos, L. Gonzalez-Guevara and O. Cauli, Autism and intellectual disability associated with mitochondrial disease and hyperlactacidemia, Int. J. Mol. Sci., 2015, 16, 3870-3884.

11 A. D. Kraft, J. M. Lee, D. A. Johnson, Y. W. Kan and J. A. Johnson, Neuronal sensitivity to kainic acid is dependent on the Nrf2-mediated actions of the antioxidant response element, J. Neurochem., 2006, 98, 1852-1865.

12 W. Wang, Y. Wu, G. Zhang, H. Fang, H. Wang, H. Zang, T. Xie and W. Wang, Activation of Nrf2-ARE signal pathway 
protects the brain from damage induced by epileptic seizure, Brain Res., 2014, 1544, 54-61.

13 W. Wang, W. P. Wang, G. L. Zhang, Y. F. Wu, T. Xie, M. C. Kan, H. B. Fang and H. C. Wang, Activation of Nrf2ARE signal pathway in hippocampus of amygdala kindling rats, Neurosci. Lett., 2013, 543, 58-63.

14 M. Mazzuferi, G. Kumar, J. van Eyll, B. Danis, P. Foerch and R. M. Kaminski, Nrf2 defense pathway: Experimental evidence for its protective role in epilepsy, Ann. Neurol., 2013, 74, 560-568.

15 Y. S. Kim, B. Podder and H. Y. Song, Cytoprotective effect of alpha-lipoic acid on paraquat-exposed human bronchial epithelial cells via activation of nuclear factor erythroid related factor-2 pathway, Biol. Pharm. Bull., 2013, 36, 802811.

16 O. Tolunay, T. Celik, M. Komur, A. E. Gezgin, M. S. Kaya and U. Celik, A rare cause of status epilepticus; alpha lipoic acid intoxication, case report and review of the literature, Eur. J. Paediatr. Neurol., 2015, 19, 730-732.

17 G. Bulaj, M. M. Ahern, A. Kuhn, Z. S. Judkins, R. C. Bowen and Y. Chen, Incorporating Natural Products, Pharmaceutical Drugs, Self-Care and Digital/Mobile Health Technologies into Molecular-Behavioral Combination Therapies for Chronic Diseases, Curr. Clin. Pharmacol, 2016, 11, 128-145.

18 H. J. Kim, W. Song, J. S. Kim, E. H. Jin, M. S. Kwon and S. Park, Synergic effect of exercise and lipoic acid on protection against kainic acid induced seizure activity and oxidative stress in mice, Neurochem. Res., 2014, 39, 15791584.

19 P. S. Santos, L. M. Campelo, R. L. Freitas, C. M. Feitosa, G. B. Saldanha and R. M. Freitas, Lipoic acid effects on glutamate and taurine concentrations in rat hippocampus after pilocarpine-induced seizures, Arq. Neuro-Psiquiatr., 2011, 69, 360-364.

20 J. Babaie, M. Sayyah, S. Choopani, T. Asgari, M. Golkar and K. Gharagozli, Toxoplasmosis accelerates acquisition of epilepsy in rats undergoing chemical kindling, Epilepsy Res., 2017, 135, 137-142.

21 O. Erbas, V. Solmaz and D. Aksoy, Inhibitor effect of dexketoprofen in rat model of pentylenetetrazol-induced seizures, Neurol. Res., 2015, 37, 1096-1101.

22 C. Palleria, A. Leo, F. Andreozzi, R. Citraro, M. Iannone, R. Spiga, G. Sesti, A. Constanti, G. De Sarro, F. Arturi and E. Russo, Liraglutide prevents cognitive decline in a rat model of streptozotocin-induced diabetes independently from its peripheral metabolic effects, Behav. Brain Res., 2017, 321, 157-169.

23 L. Zong, P. Chu, P. Huang, Y. Guo and Y. Lv, Effect of vitamin D on the learning and memory ability of FGR rat and NMDA receptor expression in hippocampus, Exp. Ther. Med., 2017, 14, 581-586.

24 M. Y. Sun, K. J. Cui, M. M. Yu, H. Zhang, X. L. Peng and H. Jiang, Bax inhibiting peptide reduces apoptosis in neonatal rat hypoxic-ischemic brain damage, Int. J. Clin. Exp. Pathol., 2015, 8, 14701-14708.
25 S. Li, L. Yang, Y. Zhang, C. Zhang, J. Shao, X. Liu, Y. Li and F. Piao, Taurine Ameliorates Arsenic-Induced Apoptosis in the Hippocampus of Mice Through Intrinsic Pathway, Adv. Exp. Med. Biol., 2017, 975, 183-192.

26 E. Shahar, U. Attias, D. Savulescu, J. Genizin, M. Gavish and R. Nagler, Oxidative stress, metalloproteinase and LDH in children with intractable and non-intractable epilepsy as reflected in salivary analysis, Epilepsy Res., 2014, 108, 117124.

27 Z. Liu, C. Yang, X. Meng, Z. Li, C. Lv and P. Cao, Neuroprotection of edaravone on the hippocampus of kainate-induced epilepsy rats through Nrf2/HO-1 pathway, Neurochem. Int., 2018, 112, 159-165.

28 K. Hashimoto, R. Majumdar and Y. Tsuji, Nuclear lamins and progerin are dispensable for antioxidant Nrf2 response to arsenic and cadmium, Cell. Signalling, 2017, 33, 69-78.

29 F. Tomaciello, K. Leclercq and R. M. Kaminski, Resveratrol lacks protective activity against acute seizures in mouse models, Neurosci. Lett., 2016, 632, 199-203.

30 J. C. Pontes, T. Z. Lima, C. M. Queiroz, S. M. Cinini, M. M. Blanco and L. E. Mello, Seizures triggered by pentylenetetrazol in marmosets made chronically epileptic with pilocarpine show greater refractoriness to treatment, Epilepsy Res., 2016, 126, 16-25.

31 P. B. McElroy, L. P. Liang, B. J. Day and M. Patel, Scavenging reactive oxygen species inhibits status epilepticus-induced neuroinflammation, Exp. Neurol., 2017, 298, 13-22.

32 E. J. Kim, R. Won, J. H. Sohn, M. A. Chung, T. S. Nam, H. J. Lee and B. H. Lee, Anti-oxidant effect of ascorbic and dehydroascorbic acids in hippocampal slice culture, Biochem. Biophys. Res. Commun., 2008, 366, 8-14.

33 C. Carrasco-Pozo, K. N. Tan and K. Borges, Sulforaphane is anticonvulsant and improves mitochondrial function, $J$. Neurochem., 2015, 135, 932-942.

34 H. S. Mohammed, Hypothermia mitigates neurochemical alterations in rat's cerebral cortex during status epilepticus induced by pilocarpine, Gen. Physiol. Biophys., 2015, 34, 425-432.

35 M. Mesgari, J. Kruger, C. T. Riemer, M. Khaleghi Ghadiri, S. Kovac and A. Gorji, Gabapentin prevents cortical spreading depolarization-induced disinhibition, Neuroscience, 2017, 361, 1-5.

36 F. da Silva Fiorin, A. P. de Oliveira Ferreira, L. R. Ribeiro, L. F. Silva, M. R. de Castro, L. R. da Silva, M. E. da Silveira Jr, A. P. Zemolin, F. Dobrachinski, S. Marchesan de Oliveira, J. L. Franco, F. A. Soares, A. F. Furian, M. S. Oliveira, M. R. Fighera and L. F. Freire Royes, The Impact of Previous Physical Training on Redox Signaling after Traumatic Brain Injury in Rats: A Behavioral and Neurochemical Approach, J. Neurotrauma, 2016, 33, 13171330.

37 M. L. Pall and S. Levine, Nrf2, a master regulator of detoxification and also antioxidant, anti-inflammatory and other cytoprotective mechanisms, is raised by health promoting factors, Acta Physiol. Sin., 2015, 67, 1-18. 
38 P. P. Trivedi and G. B. Jena, Role of alpha-lipoic acid in dextran sulfate sodium-induced ulcerative colitis in mice: studies on inflammation, oxidative stress, DNA damage and fibrosis, Food Chem. Toxicol., 2013, 59, 339-355.

39 L. Carmona-Aparicio, C. Perez-Cruz, C. Zavala-Tecuapetla, L. Granados-Rojas, L. Rivera-Espinosa, H. MontesinosCorrea, J. Hernandez-Damian, J. Pedraza-Chaverri, A. Sampieri III, E. Coballase-Urrutia and N. CardenasRodriguez, Overview of Nrf2 as Therapeutic Target in Epilepsy, Int. J. Mol. Sci., 2015, 16, 18348-18367.

40 Z. Liu, X. Yin, L. Liu, H. Tao, H. Zhou, G. Ma, L. Cui, Y. Li, S. Zhang, Z. Xu, L. Yao, Z. Cai, B. Zhao and K. Li, Association of KEAP1 and NFE2L2 polymorphisms with temporal lobe epilepsy and drug resistant epilepsy, Gene, 2015, 571, 231-236.

41 M. Nezu, N. Suzuki and M. Yamamoto, Targeting the KEAP1NRF2 System to Prevent Kidney Disease Progression, Am. J. Nephrol., 2017, 45, 473-483.

42 Y. Zhang, X. Duan, J. Li, S. Zhao, W. Li, L. Zhao, W. Li, H. Nie, G. Sun and B. Li, Inorganic Arsenic Induces NRF2-Regulated Antioxidant Defenses in Both Cerebral Cortex and Hippocampus in vivo, Neurochem. Res., 2016, 41, 2119-2128.

43 B. Wang, H. Liu, L. Yue, X. Li, L. Zhao, X. Yang, X. Wang, Y. Yang and Y. Qu, Neuroprotective effects of pterostilbene against oxidative stress injury: Involvement of nuclear factor erythroid 2-related factor 2 pathway, Brain Res., 2016, 1643, 70-79.

44 J. Wu, Y. Chen, S. Yu, L. Li, X. Zhao, Q. Li, J. Zhao and Y. Zhao, Neuroprotective effects of sulfiredoxin-1 during cerebral ischemia/reperfusion oxidative stress injury in rats, Brain Res. Bull., 2017, 132, 99-108.

45 G. Liao, R. Li, X. Chen, W. Zhang, S. Du and Y. Yuan, Sodium valproate prevents radiation-induced injury in hippocampal neurons via activation of the $\mathrm{Nrf} 2 / \mathrm{HO}-1$ pathway, Neuroscience, 2016, 331, 40-51.

46 E. C. S. Bostock, K. C. Kirkby, M. I. Garry and B. V. M. Taylor, Systematic Review of Cognitive Function in Euthymic Bipolar Disorder and Pre-Surgical Temporal Lobe Epilepsy, Front. Psychiatry., 2017, 8, 133.

47 A. K. Shetty, Hippocampal injury-induced cognitive and mood dysfunction, altered neurogenesis, and epilepsy: can early neural stem cell grafting intervention provide protection?, Epilepsy Behav., 2014, 38, 117-124.

48 K. P. Shay, A. J. Michels, W. Li, A. N. Kong and T. M. Hagen, Cap-independent Nrf2 translation is part of a lipoic acidstimulated detoxification stress response, Biochim. Biophys. Acta, 2012, 1823, 1102-1109. 